\title{
Measuring the dust content and formation in SN 1987A using detailed radiative transfer modelling
}

\author{
Maarten Baes ${ }^{1}$, Peter Camps ${ }^{1}$, Phil J. Cigan ${ }^{2}$, Christopher L. Fryer ${ }^{3}$, \\ Mikako Matsuura ${ }^{2}$ and Sam Verstocken ${ }^{1}$ \\ ${ }^{1}$ Sterrenkundig Observatorium, Universiteit Gent \\ Krijgslaan 281 S9, 9000 Gent, Belgium \\ email: maarten.baes@ugent.be, peter.camps@ugent.be, sam.verstocken@ugent.be \\ ${ }^{2}$ School of Physics and Astronomy, Cardiff University \\ The Parade, Cardiff $24 \mathrm{CF} 3 \mathrm{YB}$, UK \\ email: ciganp@cardiff.ac.uk, matsuuram@cardiff.ac.uk \\ ${ }^{3}$ Center for Theoretical Astrophysics, Los Alamos National Lab \\ Los Alamos, NM 87544, USA \\ email: fryer@lanl.gov
}

\begin{abstract}
Core-collapse supernovae are expected to be efficient producers of dust, and recent Herschel and ALMA observations have revealed up to $1 M_{\odot}$ of cold dust in the inner ejecta of SN 1987A. The formation time scale, spatial distribution and clumpiness, and the importance of the different heating sources of the dust remain poorly understood. We have started a project to make detailed 3D dust radiative transfer models for SN 1987A, based on a combination of the latest observational constraints and input from 3D hydrodynamical models and dust formation models. Preliminary results seem to indicate the need for large, micron-sized dust grains, and a relatively large dust mass.
\end{abstract}

Keywords. radiative transfer, supernovae: individual (SN 1987A)

\section{Introduction}

Detailed observations and large-scale numerical modelling efforts over the past three decades have provided much insight into SN 1987A (and into core-collapse supernovae in general). Today, SN 1987A is making the transition to a supernova remnant, as the expanding supernova is colliding with circumstellar material that was released from the star prior to the explosion. It has long been expected that core-collapse supernovae are efficient producers of dust. They provide unique physical conditions for the production of dust grains: while the average metal mass fraction in galaxies is of the order of $1 \%$, supernova ejecta can be $100 \%$ metal. Until recently, infrared observations have only detected very small amounts of warm dust in nearby supernova remnants $\left(10^{-6}\right.$ to $10^{-4} M_{\odot}$ : Gall et al. 2011). SN 1987A was the first core-collapse supernova with evidence for dust formation two years after the explosion. The reported dust masses in those early days were $10^{-6}$ to $10^{-5} M_{\odot}$ (Bouchet et al. 1991; Wooden et al. 1993).

\section{Cold dust in SN 1987A}

SN 1987A was observed in 2010 with Herschel as part of the Magellanic Cloud survey, HERITAGE, and detected at wavelengths between 100 and $350 \mu \mathrm{m}$. The dust mass 
corresponding to these observations was $0.4-0.7 M_{\odot}$, three orders of magnitude larger than previously reported (Matsuura et al. 2011). New pointed Herschel observations taken in 2012 confirmed these results, and suggest a total of $0.8 M_{\odot}$ of dust (Matsuura et al. 2015). At the same time, SN 1987A was observed and spatially resolved with ALMA between $450 \mu \mathrm{m}$ and $2.8 \mathrm{~mm}$; these observations unambiguously show the formation of 0.2 to $1 M_{\odot}$ of dust in the inner ejecta (Indebetouw et al. 2014).

One of the outstanding questions is how quickly and efficiently the dust has formed in SN 1987A (and by extension, in other core-collapse supernovae). Recent work by various teams has come to drastically different conclusions. Wesson et al. (2015) and Bevan \& Barlow (2016) used radiation transfer calculations to simulate the evolution of the observed spectral energy distribution (SED) and emission line blueshifting of SN 1987A. According to their analyses, the dust mass increases as a power law in time, and $97.5 \%$ of the dust mass formed after 1300 days. In contrast, Dwek \& Arendt (2015) applied an escape probability calculation on the same SED data, and inferred a sharply different, early and rapid dust mass evolution. According to their analysis, the dust mass had already reached $0.45 M_{\odot} 615$ days after the explosion, and it does not increase appreciably over the following two decades.

In a completely independent way, dust production in SN 1987A has been modelled from a chemical modelling perspective. In the past few years, theoretical computations of dust grain formation have become increasingly more realistic, and go far beyond the classical nucleation theory (e.g., Sarangi \& Cherchneff 2015). Sluder et al. (2016) presented a detailed analysis of dust formation in SN 1987A based on the so-called molecular nucleation theory, which tracks the abundance of each molecular species with a nonequilibrium chemical reaction network, and which includes effects such as coagulation, grain charging, evaporation, accretion, and chemical weathering. They find a grain size distribution with a power law that is steeper than the one inferred by the studies of Wesson et al. (2015) and Dwek \& Arendt (2015). Moreover, Sluder et al. (2016) predict a radically different dust composition compared to the one assumed by most previous studies.

\section{3D dust radiative transfer simulations of SN 1987A}

\subsection{SKIRT simulations}

It is clear that there are some outstanding issues related to the dust in SN 1987A: the distribution and clumpiness, the evolution of the dust production rate, the importance of the different heating sources, etc. Detailed and self-consistent dust radiative transfer simulations in a realistic setting are necessary to address these issues. Our goal is to substantially improve on the existing dust radiative transfer models for SN 1987A in several ways, by using state-of-the-art self-consistent radiative transfer techniques, and by using a combination of observational constraints and input from 3D hydrodynamical models and dust formation models. Until recently, full-scale and self-consistent dust radiative transfer calculations in 3D were almost impossible, but thanks to new techniques and increased computing power, this is possible nowadays (for a review, see Steinacker et al. 2013).

A major tool we use for our investigation is the 3D Monte Carlo radiative transfer code SKIRT (Baes et al. 2011; Camps \& Baes 2015). SKIRT has mainly been applied to investigate the dusty interstellar medium in galaxies (e.g., Baes et al. 2010; De Looze et al. 2014; Mosenkov et al. 2016), but it has also been applied to other systems including AGN accretion discs, molecular clouds, and binary systems (e.g., Stalevski et al. 2012; Hendrix 


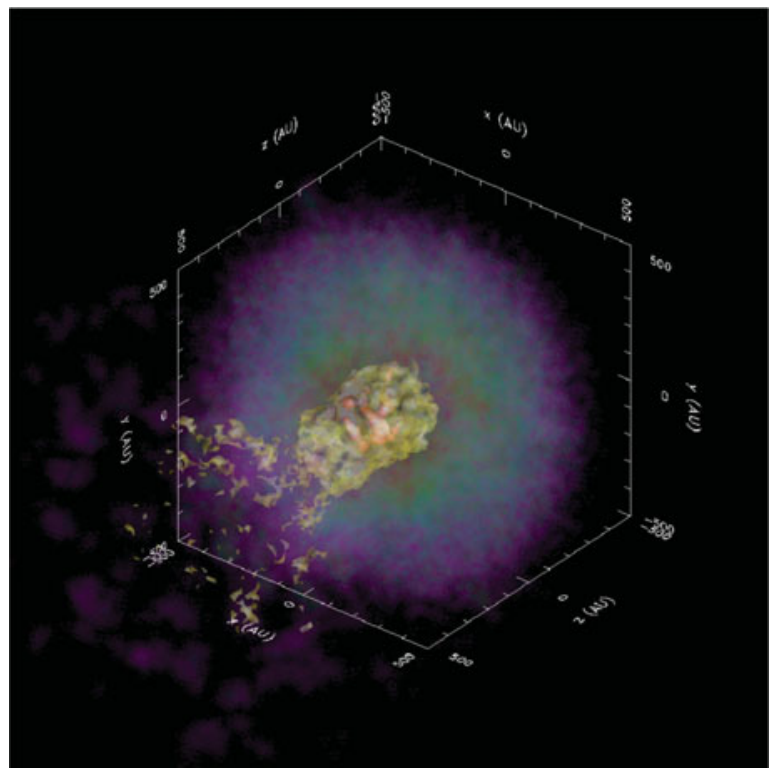

Figure 1. 3D rendering of an asymmetric core-collapse supernova explosion model from Hungerford et al. (2005), one year after the shock launch. The top-hat distribution of the imposed velocity asymmetry allows a significant portion of the cone material to expand without drag from fluid shear forces, resulting in the large splash of material at the outer ejecta. This and similar hydrodynamical models will be coupled with dust formation models and used as input for SKIRT radiative transfer simulations.

et al. 2016). One crucial aspect for the current project is that SKIRT solves the radiative transfer problem completely self-consistently, without any approximations. This implies that the effects of absorption, multiple anisotropic scattering and thermal emission (both equilibrium and transient) are taken into account. Recent work has shown there can be profound differences between such a complete 3D radiative transfer treatment and approximate solutions (Saftly et al. 2015).

\subsection{Parameterised models}

Concerning the input model for sources and dust, we use two different approaches. Our first approach is to use a (complex) parameterised model. We built a fitting routine on top of SKIRT that automatically determines the model parameters that best reproduce a given data set. The optimisation is based on genetic algorithms, which are ideally suited for fitting noisy Monte Carlo radiative transfer models (De Geyter et al. 2013, 2014). All model parameters can be varied, such as dust mass or geometry-related parameters, but also the grain size distribution, the dust clumpiness, etc. We have run initial test simulations for SN 1987A at the most recent epoch in which we vary 5 different parameters (luminosity, dust mass, silicate fraction, clumpiness and mean grain size). Preliminary results seem to indicate the need for large, micron-sized dust grains, and a relatively large dust mass $\left(>0.5 M_{\odot}\right)$, in agreement with Wesson et al. (2015).

\subsection{Models based on hydrodynamical simulations}

A more advanced approach that we will explore in the near future consists of using stateof-the-art 3D hydrodynamical simulations of core-collapse supernovae as the starting point for our radiative transfer simulations. More specifically, we will use a suite of 
models with a large variation in the progenitor star, the explosion energy and asymmetry (Fryer \& Warren 2002; Hungerford et al. 2005; Ellinger et al. 2012). These smoothed particle hydrodynamics calculations produce detailed tracers of the ejecta to calculate both detailed nucleosynthetic yields (including ${ }^{44} \mathrm{Ti}$ ) and dust formation throughout the explosion. The Lagrangian nature of these calculations (Fryer et al. 2006) allows us to resolve the shock at $10^{8}-10^{9} \mathrm{~cm}$ at the beginning of the calculation and to follow the distribution of matter out to many parsecs at late times (Figure 1). These simulations will be coupled with the molecular nucleation calculations of Sluder et al. (2016) to obtain realistic chemical compositions and size distributions for the dust grains.

We will run SKIRT radiative simulations for each of the hydrodynamical models in our suite, calculate images and SEDs for different viewpoints at different time steps in the evolution, and compare the model results with specific observations of SN 1987A. Until very recently, there were essentially no direct observational constraints on the distribution of the dust in the ejecta of SN 1987A. The latest ALMA observations, however, resolve the dust and molecular line emission in the inner ejecta (Cigan et al. 2017; Abellan et al. 2017). The comparison between the synthetic model images and the ALMA dust observations will be a strong and novel test to discriminate between the different models.

\section{Acknowledgements}

We thank the organizers for a wonderful conference in Saint-Gilles-les-Bains.

\section{References}

Abellan, F., Indebetouw, R., Matsuura, M., et al. 2017, in prep.

Baes, M., Fritz, J., Gadotti, D. A., et al. 2010, A\&A, 518, L39

Baes, M., Verstappen, J., De Looze, I., et al. 2011, ApJS, 196, 22

Bevan, A. \& Barlow, M. J. 2016, MNRAS, 456, 1269

Bouchet, P., Phillips, M. M., Suntzeff, N. B., et al. 1991, AESA, 245, 490

Camps, P. \& Baes, M. 2015, Astronomy and Computing, 9, 20

Cigan, P., Matsuura, M., Gomez, H. L., et al. 2017, in prep.

De Geyter, G., Baes, M., Fritz, J., \& Camps, P. 2013, A\&A A, 550, A74

De Geyter, G., Baes, M., Camps, P., et al. 2014, MNRAS, 441, 869

De Looze, I., Fritz, J., Baes, M., et al. 2014, A\&GA, 571, A69

Dwek, E. \& Arendt, R. G. 2015, ApJ, 810, 75

Ellinger, C. I., Young, P. A., Fryer, C. L., \& Rockefeller, G. 2012, ApJ, 755, 160

Fryer, C. L. \& Warren, M. S. 2002, ApJL, 574, L65

Fryer, C. L., Rockefeller, G., \& Warren, M. S. 2006, ApJ, 643, 292

Gall, C., Hjorth, J., \& Andersen, A. C. 2011, A\&AR, 19, 43

Hendrix, T., Keppens, R., van Marle, A. J., et al. 2016, MNRAS, 460, 3975

Hungerford, A. L., Fryer, C. L., \& Rockefeller, G. 2005, ApJ, 635, 487

Indebetouw, R., Matsuura, M., Dwek, E., et al. 2014, ApJL, 782, L2

Matsuura, M., Dwek, E., Meixner, M., et al. 2011, Science, 333, 1258

Matsuura, M., Dwek, E., Barlow, M. J., et al. 2015, ApJ, 800, 50

Mosenkov, A. V., Allaert, F., Baes, M., et al. 2016, A\&A, 592, A71

Saftly, W., Baes, M., De Geyter, G., et al. 2015, A\& $A$, 576, A31

Sarangi, A. \& Cherchneff, I. 2015, A $\mathscr{G} A, 575$, A95

Sluder, A., Milosavljevic, M., \& Montgomery, M. H. 2016, arXiv:1612.09013

Stalevski, M., Fritz, J., Baes, M., Nakos, T., \& Popović, L. Č. 2012, MNRAS, 420, 2756

Steinacker, J., Baes, M., \& Gordon, K. D. 2013, ARA\&A, 51, 63

Wesson, R., Barlow, M. J., Matsuura, M., \& Ercolano, B. 2015, MNRAS, 446, 2089

Wooden, D. H., Rank, D. M., Bregman, J. D., et al. 1993, ApJS, 88, 477 